\title{
Assessment organic production of kyuri farms focuses on microbiological contamination with good agricultural practices (GAP) standard criteria.
}

\author{
Okta Pringga Pakpahan ${ }^{1 *}$, Kenji Hosono², and Hiroyuki Nakaki ${ }^{2}$ \\ 1) Food science and technology department, Agriculture and Animal Science Faculty, \\ Muhammadiyah University of Malang, Indonesia \\ 2) Graduate School of Biosphere Science, Hiroshima University, Japan.
}

*corresponding author: okapringga@umm.ac.id

\begin{abstract}
The assessment was based on interviews result and experiment laboratory assay with the three organic kyuri farmers in Saga City focus prevention activities of microbiological contamination. Each farm the 21 samples (soil, kyuri seedlings, natural fertilizer, and water resource) were taken during six times visited or one cultivation production cycle. The object was a focus on hygiene attribute (coliforms) and safety (Salmonella spp and E. coli O157: H7), the evaluation activities of farmers' food safety practice used GAP standard as an indicator. The laboratory results in both of three kyuri farmers, coliforms ranging from 3.0 to $4.7 \mathrm{log} 10 \mathrm{cfu} / \mathrm{g}$, E.coli O157: H7 was detected only at the farm 3 and Salmonella spp was not detected. The evaluating activities through self-assessment tool describe all three farms have two major hazards focus, one is chemical control such as synthetic substances uses, pesticide residue and chemical contaminations, two is a microbial hazard due to contact with raw material, and natural characteristics. The overall study, the farmers in the middle level of application food safety practice.
\end{abstract}

Keywords: GAP, Microbiology, Farmers

\section{Introduction}

In recently, consumptions of global organic fresh produce have increased with major reason personal preference, healthy, and sustainable food production (FAO, 2012). The organic products should be free from synthetic and chemical substances during planting to post-harvest process (Aquino \& Assis, 2007). As a natural characteristic of organic produce is more susceptible to spreading microbiology contaminations, some literature has been increasing concern in microbiological quality of organic produce (Delaquis, Bach, \& Dinu, 2007; Oliveira, et al., 2010). In Japan, Japanese cucumber "Kyuri" have used in a diverse processing way on local food and have a huge percentage of organic fresh produce consumption (MAFF, 2016). However, Kyuri more prone to contamination of microbiological due to the soil environment and peel provides potential conditions for fungal growth (Alam, et al., 2015).

The Pathogenic contaminations and fungal growth of fresh produce have frequently through from soil during preparation field, natural fertilizers are not mature when it using, water supply is contaminated with other water resources. (Codex, 2005; Oliveira et al., 2010; Umesha et al., 2017). Another aspect, such as sophisticated facilities and equipment, adequate sanitary conditions and packaging are of utmost 
importance attribute to assure the products are not contaminated along the production system.

The data related to microbiological contamination issue in Japan is not specific to organic kyuri consumptions. Due to that, the purpose of our study was to investigate the microbiological preventing activities and food safety management aspect along the production. The assessment and indicator is base on good agricultural practices attribute (GAP). Three organic kyuri farms in Hiroshima Prefecture were selected and visited six times for observations and take a sampling for microbiological contamination assay (Salmonella spp, E. coli O157:H7 and coliforms) during the kyuri production systems. At last visit, comprehensive assessment using a standardized self-assessment questionnaire develop by Lunning, et al., 2011, Kirezieva et al., 2013, and Uyttendaele et al., 2010 is applied to asses level of food safety activities.

\section{Material and Methods \\ Microbiological sampling test}

Critical sampling locations (CSLs) was used to identifying bottlenecks in microbial contaminations preventions. In line with that, $10 \mathrm{CSL}$ (table 1) has modified base on potential risk factors (i.e., soil, water, natural fertilizer, and product handling) could contribute to microorganism growth (Yeni et al., 2016). Which sampling plan was a focus to obtain coliforms as hygiene attribute, and both E. coli O157: H7 and Salmonella spp as food safety attribute. Visit and sampling was conducted from May 2017 until October 2017 with a total of 6 times visited. First visit, one week before cultivation preparation, second visit, on the planting day, third visit, three weeks after planting a day, fourth visit, two weeks before harvest, fifth visit, at harvest day and last visit before shipping. This step was repeated at the respective farms (Table 1 and 2).

\section{Sampling method}

Samplings are divided into two aspects, first, aspect the natural material (soil, natural fertilizer, water resource and kyuri seedling) and second, aspect preparation in post-harvest activities (washing treatment, cutting and grading step and transport container box). At each activities step, samples were collected during visiting the farms. In specifically, aspect the natural material, for material soil and natural fertilizer were collected $100 \mathrm{~g}$ on the top part of each ground field and composting locations. The samples were stored in sterile plastic bags for advance microbiological analyses in a laboratory. At water resource step, $10 \mathrm{~L}$ was pumped to sterile gallon from water tank. The kyuri seedlings were pooled using a seedling tray. Furthermore, post-harvest activities samples were collected using sterilized swabs rubbed two-way streak directions delimited in square $2 \mathrm{~cm}^{2} \times 2 \mathrm{~cm}^{2}$ on a final product at washing treatment, cutting and grading step and transport container box activities. To keep moistened for the microbial growth the test tubes are containing $0.1 \%$ peptone water placed in thermal boxes and store in refrigeration $\left(7^{\circ} \mathrm{C}\right)$. 


\section{Microbiological analysis methods}

Table 2 has presented a method and indicators are used to assess the degree of hygiene and safety attribute in each farm. First, at hygiene attribute is used amount coliforms to analyse the quality level of the sanitation program applied. Second, at food safety attribute is used total plate count colonies describe in ISO 16140:2010 (ISO, 2010) to analyse the level of the contamination at each activity from E. coli O157: H7 and Salmonella spp. The validation characteristic of colonies using a gram stain test method on selective media agar $38^{\circ} \mathrm{C}$ and growth at the presence of $4.0 \% \mathrm{NaCl}$.

Step first to analyses hygiene attribute, $1 \mathrm{~g}$ samples took from natural materials has collected placed in a test tube with $9 \mathrm{ml}$ water. The samples were homogenized in a digital vortex mixer (cole-parmer) for $60 \mathrm{~s}$ with $2.000 \mathrm{rpm}$. Afterwards, the solution used for decimal dilutions until $10^{4}$ and took $1 \mathrm{ml}$ using pour plate method into a petri dish containing nutrient agar Merck (Darmstadt, Germany) and incubated for $48 \mathrm{~h}$ at $37^{\circ} \mathrm{C}$, this is prepared in triplicate. However, to analyse water samples using the Most Probable Number (MPN) method which $50 \mathrm{ml}$ water samples filled into the multiple-tube technique (Standard Methods for Examination of Water and Wastewater, 1998).

\section{Questionnaire self-assessment the degree of safety base on good agricultural practices (GAP) standard}

The self-assessment tools used a four-set questionnaire to get insight from the interview during the visited farm. These questionnaire designed focus on prevention or control activities of contamination or growth microbiological with a total of 28 indicators required by GAP standard. For each indicator has three levels of assessment low-risk, medium or high-risk level and data will analysis with the statistical method (Jacxsens et al., 2013; Kirezieva et al., 2015). The interviewees are individual have responsible for each activity in the indicator.

The first set of the questionnaire examining management operation of farms through context factors described from performance cultivation process characteristics and orientations of the organization. The second set of the questionnaire cross-examines monitoring activities done by farms have to meet with GAP standard through two aspects performance of monitoring activities and monitoring activities operation. The third set of the questionnaire verification the indicators during the assurance activities. The fourth set of the questionnaire analyzing the system output through the report feedback or complaints.

\section{Result and Discussion}

Table 3 presents a result from 63 samples (21 per farm) while table 4 demonstrates results irrigation water assay. Overall both results of three kyuri farmers, coliforms ranging from 3.0 to $4.7 \log 10 \mathrm{cfu} / \mathrm{g}$, E.coli $\mathrm{O} 157: \mathrm{H} 7$ was detected only at the farm 3 
and Salmonella spp was not detected. Water samples collected in the tank was not detected coliforms in all farms. Moreover, in all farms, examined counts have decreased when approaching to post-harvest. For example, coliform started (T0) 3.5 to 4.7 (T1), 3.5 to 4.2 (T2) 3.4 to 4.1 (T3) 3.4 to 3.9 (T4) 3.3 to 3.7 and (T5) 2.0 to 3.3.

The highlight bold activites observed during the sampling time is hygiene (transport boxes and hands of workers) and safety (soil, natural fertilizer, and kyuri seedlings). These activities are the critical control point from the whole process, all of the kyuri farms presented low microbiological contamination. Moreover, washing used a secure water tank supplies was a tendency of decreasing the microbial potential contamination. The final kyuri samples from all farms meet with a Japanese regulation that maximum coliforms $10 \times 2 \mathrm{cfu} / \mathrm{g}$, E.coli O157: H7 and Salmonella spp should negative. However, the presence of E.coli O157: H7 was detected only in natural fertilizer at the farm 3, due to failing decomposition of pathogens period. In line with that, study of Johannessen (2005) findings E.coli O157: H7 is a serious problem which may lead contamination in the irrigation system or spreadings to other kyuri fruits since it is served or eaten in raw.

Another major indicator that can be linked to the microbiological potential contamination is a water resource. The results are ranging from 6.3 to $20 \mathrm{MPN} / \mathrm{ml}$ at all times, it means the potential is negative. All the farms obtained the main resource for water is pumping from a well and filtered before depositing to a huge tank. Afterwards, the tank is channelled to pipe network for a sprinkle and faucet. With this system, the irrigation be sufficient to eliminate a contamination potential to avoid using standstill water or pond (Olaimat, et al., 2012).

The evaluating activities through self-assessment tool describe all three farms have two major hazards focus, one is chemical control such as synthetic substances uses, pesticide residue and chemical contaminations, two is a microbial hazard due to contact with raw material, and natural characteristics. In table 5 part I demonstrated that three farms proved operated in high performance of microbial risk. Due to cultivation in the same region and environment, all of the farmers attending organic production practices that were provided by cooperative and food safety authorities and each activity is performed to GAP criteria guidelines. Others, during the interviews the workers have high commitment concerned with quality improvements. 
Table 1. Timeline of selected critical sampling locations (CSL). T0: field preparations; T1: Start of the planting; T2: After planting two weeks; T3: After planting four weeks T4: Two weeks before harvest; T5: Harvesting T6:

Postharvest handling.

\begin{tabular}{|c|c|c|c|c|c|c|c|c|c|c|c|}
\hline \multicolumn{2}{|r|}{ To } & \multicolumn{2}{|r|}{$\mathrm{T} 1$} & \multicolumn{2}{|r|}{$\mathrm{T} 2$} & \multicolumn{2}{|r|}{ T3 } & \multicolumn{2}{|r|}{$\mathrm{T} 4$} & \multicolumn{2}{|r|}{ T5 } \\
\hline CSL 1 & Manure & CSL 5 & Manure Soil & CSL 5 & Manure Soil & CSL 5 & Manure Soil & CSL 5 & Manure Soil & CSL 5 & Manure Soil \\
\hline SL 2 & manure Soil & CSL 6 & Kyuri & CSL 6 & Kyuri & CSL 6 & Kyuri & CSL 6 & Kyuri & CSL 6 & Kyuri \\
\hline CSL 3 & $\begin{array}{l}\text { Seedlings in } \\
\text { soil }\end{array}$ & CSL 10 & $\begin{array}{l}\text { Irrigation } \\
\text { Water Tank }\end{array}$ & CSL 10 & $\begin{array}{l}\text { Irrigation } \\
\text { Water Tank }\end{array}$ & CSL 10 & $\begin{array}{l}\text { Irrigation } \\
\text { Water Tank }\end{array}$ & CSL 10 & $\begin{array}{l}\text { Irrigation } \\
\text { Water Supply }\end{array}$ & CSL 7 & $\begin{array}{l}\text { Kyuri after } \\
\text { washing }\end{array}$ \\
\hline CSL 4 & Seedlings & CSL 10 & $\begin{array}{l}\text { Irrigation } \\
\text { Water supply }\end{array}$ & CSL 10 & $\begin{array}{l}\text { Irrigation } \\
\text { Water supply }\end{array}$ & CSL 10 & $\begin{array}{l}\text { Irrigation } \\
\text { Water supply }\end{array}$ & CSL 10 & $\begin{array}{l}\text { Irrigation } \\
\text { Water supply }\end{array}$ & CSL 8 & $\begin{array}{l}\text { Swab Farmer } \\
\text { Hands }\end{array}$ \\
\hline CSL 10 & $\begin{array}{l}\text { Irrigation } \\
\text { Water supply }\end{array}$ & & & & & & & & & CSL 9 & $\begin{array}{l}\text { Swab of } \\
\text { transport Box } \\
\text { of kyuri }\end{array}$ \\
\hline
\end{tabular}

Table 2. Identification step in microbiological methodologies sampling of critical sampling locations (CSL)

\begin{tabular}{|c|c|c|c|c|c|c|}
\hline $\mathrm{CSL}$ & Samples & $\begin{array}{l}\text { Amount of } \\
\text { sampling }\end{array}$ & Period & Parameters & Method & Peformance of the result \\
\hline 1 & Manure & 4 Samples & T0 & $\begin{array}{l}\text { Coliforms } \\
\text { Salmonella } \\
\text { E.Coli O157:H7 }\end{array}$ & $\begin{array}{l}\text { ISO 16140:2010, AOAC } \\
(2002) \\
\text { ISO } 16140: 2010\end{array}$ & $\begin{array}{l}2.000 \mathrm{NPM} / \mathrm{g} \\
\mathrm{A} / 25 \mathrm{~g} \\
\mathrm{~A} / 25 \mathrm{~g}\end{array}$ \\
\hline 2 & Manured Soil & 9 Pooled Samples & T0 & $\begin{array}{l}\text { Coliforms } \\
\text { Salmonella } \\
\text { E.Coli O157:H7 }\end{array}$ & $\begin{array}{l}\text { ISO 16140:2010, AOAC } \\
(2002) \\
\text { ISO 16140:2010 }\end{array}$ & $\begin{array}{l}2.000 \mathrm{NPM} / \mathrm{g} \\
\mathrm{A} / 25 \mathrm{~g} \\
\mathrm{~A} / 25 \mathrm{~g}\end{array}$ \\
\hline 3 & Seedlings in Soil & 3 Pooled Samples & T0 & $\begin{array}{l}\text { Coliforms } \\
\text { Salmonella } \\
\text { E.Coli O157:H7 }\end{array}$ & $\begin{array}{l}\text { ISO 4832:2006, AOAC } \\
(2002) \\
\text { ISO 16140:2010 }\end{array}$ & $\begin{array}{l}10^{2} \\
\mathrm{~A} / 25 \mathrm{~g} \\
\mathrm{~A} / 25 \mathrm{~g}\end{array}$ \\
\hline 4 & Seedling & 1 Sample & $\mathrm{T} 0$ & Coliforms & $\begin{array}{l}\text { ISO 16140:2010, AOAC } \\
(2002)\end{array}$ & $10^{2}$ \\
\hline 5 & Manured Soil & 9 Pooled Samples & $\begin{array}{l}\mathrm{T} 1 \\
\mathrm{~T} 2 \\
\mathrm{~T} 3 \\
\mathrm{~T} 4 \\
\mathrm{~T} 5\end{array}$ & $\begin{array}{l}\text { Coliforms } \\
\text { E.Coli O157:H7 } \\
\text { Salmonella }\end{array}$ & $\begin{array}{l}\text { ISO } 16140: 2010, \text { AOAC } \\
(2002) \\
\text { ISO } 16140: 2010\end{array}$ & $\begin{array}{l}10^{2} \\
\mathrm{~A} / 25 \mathrm{~g} \\
\mathrm{~A} / 25 \mathrm{~g}\end{array}$ \\
\hline 6 & Kyuri & 9 Pooled Samples & $\mathrm{T} 5$ & $\begin{array}{l}\text { Coliforms } \\
\text { Salmonella }\end{array}$ & $\begin{array}{l}\text { ISO 16140:2010, AOAC } \\
(2002)\end{array}$ & $\begin{array}{l}10^{2} \\
\mathrm{~A} / 25 \mathrm{~g}\end{array}$ \\
\hline
\end{tabular}


Swab of farmers hands

9

10 container of Kyuri Irrigation water supply

E.Coli O157:H7

Coliforms

Salmonella

E.Coli O157:H7

$10 \times 50 \mathrm{~cm}^{2}$

$200 \mathrm{ml}$
Coliforms

T5

T5

Coliforms

Coliforms

Salmonella

E.Coli O157:H7
ISO 16140:2010

ISO 16140:2010, AOAC

(2002)

ISO 16140:2010

ISO 16140:2010, AOAC (2002)

ISO 16140:2010, AOAC (2002)

$20^{\text {th }}$ APHA (1998)

ISO 16654:2001
A/25g

$10^{2}$

$\mathrm{A} / 25 \mathrm{~g}$

$\mathrm{A} / 25 \mathrm{~g}$

$\leq 0.6 \log \mathrm{cfu} / 25 \mathrm{~cm}^{2}$

(below detection)

$\leq 0.6 \log \mathrm{cfu} / 25 \mathrm{~cm}^{2}$

(below detection)

$2 \times 10^{2} \mathrm{cfu} / 200 \mathrm{ml}$

$\mathrm{A} / 25 \mathrm{ml}$

$\mathrm{A} / 25 \mathrm{ml}$

Table 3. Results of microbial samples assay at three organic kyuri farms.

\begin{tabular}{|c|c|c|c|c|c|c|c|c|c|c|c|c|}
\hline \multirow[b]{2}{*}{ Visit } & \multirow[b]{2}{*}{ CSL } & \multirow[b]{2}{*}{ Samples } & \multirow[b]{2}{*}{$\begin{array}{l}\text { Amunutu ve } \\
\text { sampling }\end{array}$} & \multirow{2}{*}{$\begin{array}{l}\text { Farm } 1 \\
\text { Coliforms }\end{array}$} & \multicolumn{5}{|c|}{ Farm 2} & \multicolumn{3}{|l|}{ Farm 3} \\
\hline & & & & & $\begin{array}{l}\text { E.Coli } \\
\text { O157:H7 }\end{array}$ & Salmonella & Coliforms & $\begin{array}{l}\text { E.Coli } \\
\text { O157:H7 }\end{array}$ & Salmonella & Coliforms & $\begin{array}{l}\text { E.Coli } \\
\text { O157:H7 }\end{array}$ & Salmonella \\
\hline T0 & 1 & Manure & 3 & $3.5( \pm 0.11)$ & $-(0 / 2)$ & $-(0 / 3)$ & $5.5( \pm 0.92)$ & $-(0 / 2)$ & $+(2 / 3)$ & $4.5( \pm 0.33)$ & $+(1 / 2)$ & $-(0 / 3)$ \\
\hline T0 & 2 & Manured Soil & 9 & $3.4( \pm 0.70)$ & $-(0 / 3)$ & $-(0 / 3)$ & $4.0( \pm 0.13)$ & $-(0 / 2)$ & $+(2 / 3)$ & $4.7( \pm 0.55)$ & $-(0 / 3)$ & $-(0 / 3)$ \\
\hline T0 & 3 & Seedling in soil & 3 & 4.5 & $-(0 / 1)$ & $-(0 / 1)$ & 5.7 & $-(0 / 1)$ & $-(0 / 1)$ & 4.0 & $-(0 / 1)$ & $-(0 / 1)$ \\
\hline T0 & 4 & Seedling & 1 & 3.5 & $-(0 / 1)$ & $-(0 / 1)$ & 4.3 & $-(0 / 2)$ & $-(0 / 2)$ & 4.2 & $-(0 / 1)$ & $-(0 / 1)$ \\
\hline $\mathrm{T} 1$ & 5 & Manured Soil & 9 & $3.5( \pm 0.67)$ & $-(0 / 1)$ & $-(0 / 1)$ & $3.9( \pm 0.33)$ & $-(0 / 1)$ & $-(0 / 1)$ & $4.2( \pm 0.92)$ & $-(0 / 3)$ & $-(0 / 3)$ \\
\hline $\mathrm{T} 2$ & 5 & Manured Soil & 9 & $3.4( \pm 0.07)$ & $-(0 / 3)$ & $-(0 / 3)$ & $4.1( \pm 0.44)$ & $-(0 / 3)$ & $-(0 / 3)$ & $4.2( \pm 0.30)$ & $-(0 / 3)$ & $-(0 / 3)$ \\
\hline T3 & 5 & Manured Soil & 9 & $3.4( \pm 0.72)$ & $-(0 / 3)$ & $-(0 / 3)$ & $3.6( \pm 0.73)$ & $-(0 / 3)$ & $-(0 / 3)$ & $3.9( \pm 1.53)$ & $-(0 / 3)$ & $-(0 / 3)$ \\
\hline $\mathrm{T} 4$ & 5 & Manured Soil & 9 & $3.3( \pm 1.34)$ & $-(0 / 3)$ & $-(0 / 3)$ & $3.7( \pm 0.92)$ & $-(0 / 3)$ & $-(0 / 3)$ & $3.5( \pm 0.66)$ & $-(0 / 3)$ & $-(0 / 3)$ \\
\hline T5 & 5 & Manured Soil & 9 & $3.3( \pm 0.78)$ & $-(0 / 3)$ & $-(0 / 3)$ & $3.6( \pm 1.03)$ & $-(0 / 3)$ & $-(0 / 3)$ & $3.1( \pm 0.93)$ & $-(0 / 3)$ & $-(0 / 3)$ \\
\hline T5 & 6 & Kyuri & 9 & $3.3( \pm 1.83)$ & $-(0 / 3)$ & $-(0 / 3)$ & $3.5( \pm 0.41)$ & $-(0 / 3)$ & $-(0 / 3)$ & $3.0( \pm 1.23)$ & $-(0 / 3)$ & $-(0 / 3)$ \\
\hline T5 & 7 & $\begin{array}{l}\text { Kyuri after } \\
\text { washing }\end{array}$ & 9 & $3.2( \pm 0.17)$ & $-(0 / 3)$ & $-(0 / 3)$ & $3.3( \pm 1.63)$ & $-(0 / 3)$ & $-(0 / 3)$ & $3.0( \pm 0.37)$ & $-(0 / 3)$ & $-(0 / 3)$ \\
\hline T5 & 8 & Worker hands & 9 & $3.1( \pm 0.62)$ & $-(0 / 3)$ & $-(0 / 3)$ & $2.9( \pm 0.93)$ & $-(0 / 3)$ & $-(0 / 3)$ & $2.2( \pm 1.21)$ & $-(0 / 3)$ & $-(0 / 3)$ \\
\hline T5 & 9 & boxes & 9 & $3.0( \pm 0.89)$ & $-(0 / 3)$ & $-(0 / 3)$ & $2.0( \pm 0.06)$ & $-(0 / 3)$ & $-(0 / 3)$ & $2.9( \pm 0.63)$ & $-(0 / 3)$ & $-(0 / 3)$ \\
\hline
\end{tabular}


Table 4. Results Irrigation water assay at three organic kyuri farms.

\begin{tabular}{|c|c|c|c|c|c|c|c|c|c|c|c|c|}
\hline \multirow[b]{2}{*}{ Visit } & \multirow[b]{2}{*}{ CSL } & \multirow[b]{2}{*}{ Samples } & \multirow[b]{2}{*}{$\begin{array}{l}\text { rumvunic vi } \\
\text { sampling }\end{array}$} & \multicolumn{3}{|l|}{ Farm 1} & \multicolumn{3}{|l|}{ Farm 2} & \multicolumn{3}{|l|}{ Farm 3} \\
\hline & & & & $\begin{array}{l}\text { Coliforms } \\
\text { NPM/200ml }\end{array}$ & $\begin{array}{l}\text { E.Coli } \\
\text { O157:H7 } \\
\text { NPM/25ml }\end{array}$ & $\begin{array}{l}\text { Salmonella } \\
\text { NPM/25ml }\end{array}$ & $\begin{array}{l}\text { Coliforms } \\
\text { NPM/200ml }\end{array}$ & $\begin{array}{l}\text { E.Coli } \\
\text { O157:H7 } \\
\text { NPM/25ml }\end{array}$ & $\begin{array}{l}\text { Salmonella } \\
\text { NPM/25ml }\end{array}$ & $\begin{array}{l}\text { Coliforms } \\
\text { NPM/200ml }\end{array}$ & $\begin{array}{l}\text { E.Coli } \\
\text { O157:H7 } \\
\text { NPM/25ml }\end{array}$ & $\begin{array}{l}\text { Salmone } \\
\text { NPM/25 }\end{array}$ \\
\hline T0 & 1 & $\begin{array}{l}\text { Irrigations } \\
\text { water tank }\end{array}$ & 1 & 14 & absence & absence & 18 & absence & absence & 20 & absence & absence \\
\hline $\mathrm{T} 1$ & 2 & $\begin{array}{l}\text { Irrigations } \\
\text { water tank }\end{array}$ & 1 & 13 & absence & absence & 18 & absence & absence & 20 & absence & absence \\
\hline $\mathrm{T} 2$ & 3 & $\begin{array}{l}\text { Irrigations } \\
\text { water tank }\end{array}$ & 1 & 13 & absence & absence & 11 & absence & absence & 20 & absence & absence \\
\hline T3 & 4 & $\begin{array}{l}\text { Irrigations } \\
\text { water tank }\end{array}$ & 1 & 10 & absence & absence & 7.1 & absence & absence & 17 & absence & absence \\
\hline $\mathrm{T} 4$ & 5 & $\begin{array}{l}\text { Irrigations } \\
\text { water tank }\end{array}$ & 1 & 8.7 & absence & absence & 6.3 & absence & absence & 16 & absence & absence \\
\hline
\end{tabular}

Table 5. Self-assessment result degree based on good agricultural practices (GAP) standard attribute

\begin{tabular}{|c|c|c|c|c|}
\hline Attribute Descripsions & $\begin{array}{l}\text { Assumption linked to indicator (based on Kirezieva, Jacxsens et al., 2013, } \\
\text { Kirezieva, Nanyunja et al., 2013) }\end{array}$ & Farm 1 & Farm 2 & $\overline{\text { Farm }}$ \\
\hline \multicolumn{5}{|c|}{ PART I. Context factors } \\
\hline \multicolumn{5}{|c|}{ Cultivation process characteristics } \\
\hline $\begin{array}{l}\text { Risk of raw materials } \\
\text { (microbial) }\end{array}$ & $\begin{array}{l}\text { Raw materials used in kyuri seedlings that can lead to microbial } \\
\text { contamination due to natural characteristics of materials are concern } \\
\text { during cultivation practices step and follow GAP requirement criteria }\end{array}$ & 3 & 3 & 3 \\
\hline $\begin{array}{l}\text { Risk of final product } \\
\text { (microbial) }\end{array}$ & $\begin{array}{l}\text { Kyuri surface are prone to pathogen or fungal growth, final treatment such } \\
\text { microbiological hygiene or pathogen prevalence follow GAP requirement } \\
\text { criteria increase food safety performance }\end{array}$ & 3 & 3 & 3 \\
\hline Production system & $\begin{array}{l}\text { Open field cultivation system contact with the environments and the soils } \\
\text { that can minimalize risk to microbial contamination follow GAP } \\
\text { requirement criteria which less direct. }\end{array}$ & 3 & 3 & 3 \\
\hline Water supply & $\begin{array}{l}\text { Water supplies tank should prevent contamination others water resourche } \\
\text { likelihoods (i.e., rainwater, uncontrolled surface water, water from ponds) } \\
\text { and treatment follow GAP requirement criteria }\end{array}$ & 3 & 3 & 3 \\
\hline Mean Cultivation process characteristics & & 3.0 & 3.0 & 3.0 \\
\hline \multicolumn{5}{|c|}{ Organization and chain } \\
\hline Presence of technological staff & $\begin{array}{l}\text { Farms having internal expertise staff with technology technician support in } \\
\text { food safety attribute follow GAP requirement criteria. }\end{array}$ & 2 & 3 & 3 \\
\hline
\end{tabular}


Variability in workforce composition

Sufficiency of workers competences

Extent of management commitment

Degree of employee involvement

Sufficiency supporting information systems

Severity of stakeholder's requirements

Extent of power in supplier relationships

Food safety information exchange

Logistic facilities

Inspections of food safety authorities

Specific external support

Specific legislation
Workforce composition variability $20 \%$ part-time and $80 \%$ permanent workers which rotation is limited with specific safety tasks at cultivation process step attribute follow GAP requirement criteria.

Hiring technical, operators and supervisior having specific skill, training aspect and education in food hygiene and safety attribute follow GAP requirement criteria.

Management very eager commitment improving on food safety control system following adequately improving workers issues solving (i.e., welfare) follow GAP requirement criteria.

Workers attending machine operation instructions workshop, hygine and safety attribute traning lead to improving motivated and skills in commitment follow GAP requirement criteria.

Information systems such as book record, or computerize consist of cultivation activity, hygine assesment, safety tasks and assurance activities in real time activity record requiring follow GAP requirement criteria

Stakeholders (association organizations, government, retailers, wholesalers) requird differing requirements, selective, sophisticated, higyne and safety easy monitoring by computerized follow GAP requirement criteria.

Incoming materials from supplier requiring attribute such update, advance and traceable which higyne and safety monitoring under control supplier follow GAP requirement criteria

Sophisticated information sharing system must deal with all recording data of hygiene and ssafety activity follow GAP requirement criteria.

Logistic facilities should have adequate and strictly maintaining to preventing microorganisms and contamination on environmental facilities which is follow GAP requirement criteria.

Inspections system by authorities food safety control body should adequate and give properly feedback for improving perofmanc of the hygiene and safety activity follow GAP requirement criteria

External specific support on production system should giving adequate

consider such as opinion, observation and assesment related hygiene and safety attribute may improving the food safety activity decisions follow GAP requirement criteria.

Farms adopting detailed national food legislation on food hygiene and safety attribute adequate following with GAP requirement criteria. 
Hygienic design of equipment and facilities

Maintenance and calibration program

Storage facilities

Sanitation program(s)

Personal hygiene requirements

Incoming material monitoring

Packaging materials

Supplier monitoring

Organic fertilizer program

Irrigation method

Analytical methods to assess pathogens

Sampling plan for microbial assessment

\section{Mean Performance of Monitoring} activities

Actual availability of procedures

The actuality of compliance to procedures
Facilities designs focus on critical hygiene and equipment considering efficient cleaning and preventing cross-contamination following with GAP requirement criteria.

The maintenance program should have schedule period and specific manual guide depent on machine follow with GAP requirement criteria. Storage facilities designs for keep humidity, air circulation and temperature contidions to preventing cross-contamination or microorganisms' growth follow GAP requirement criteria.

Sanitation facilities should have adequate equipment for cleaning period following specific aspect instructions to preventing cross-contamination or microorganisms growth follow GAP requirement criteria.

Farms should have specific personal hygiene guidance to prevent contamination follow GAP requirement criteria.

Farm should have incoming material monitoring record and verifications before using the material in every single step follow GAP requirement criteria.

Packaging materials using compatible material product friendly to preventing product harm or damange follow GAP requirement criteria. More specific criteria and systematic evaluation process for selecting supplier material follow GAP requirement criteria.

Adequate facilities and equipment to support organic fertilizer programs follow GAP requirement criteria.

Irrigation methods should have limited direct contact among water and edible parts to preventing contamination follow GAP requirement criteria.

More adequed rapid pathogen identification methods fullil GAP requirement criteria.

Periodical sampling data program on real time product and treatment activities information follow GAP requirement criteria.

\section{Monitoring activities operation}

Accesable of traceability procedures and programing follow GAP
requirement criteria.

The detail activities at all steps record in computerized lead to compliance behavior in monitoring follow GAP requirement criteria. 
Actual hygienic performance of equipment and facilities

Actual storage/cooling capacity

Actual process capability of packaging

Actual performance of analytical

equipment

Mean monitoring activities operation

Validation of preventive measures

Verification of equipment and methods related performance

Documentation system

Record-keeping system

Mean assurance activities

Equipment and facilities should mainaining hygienic performance follow GAP requirement criteria.

Storage/cooling facilities should have stable temperature to reduce risk of contaminations follow GAP requirement criteria.

Consistent packaging product material base on the suitability product

follow GAP requirement criteria.

Periodicals calibrations equipment program to avoid incompatibility measument during analysis activities follow GAP requirement criteria

\section{PART III. Assurance activities}

A laboratory evidance and verification observation base preventive measure act taken follow GAP requirement criteria.

Competence verificated from the third part follow GAP requirement criteria.

All information and documen have data base which is support for sharing to other stakholders follow GAP requirement criteria.

Computerized record system on real time with documentation at every step follow GAP requirement criteria.

Evaluation of good agricultural practices

Seriousness of remarks

Hygiene-related and microbiological food safety

Product sampling for microbiological performance

Judgment criteria for microbiological results

Non-conformities

Mean assurance activities

\section{PART IV. System output}

Inspection and evaluation current applications which auditing by competence organization body follow GAP requirement criteria.

All inditaros in good or excellent marking by competence organization body follow GAP requirement criteria.

Complaints or feedback regarding hygiene and safety product issues in small number.

Microbiological performance measuring with adequate methods with accurate result follow GAP requirement criteria.

Considering thrid party analyses to assement the microbiological performance follow GAP requirement criteria.

All non-conformities indicator at low level or good or excellent marking follow GAP requirement criteria.

2.5

2.5

2.4

3

3

2.7
3

3

3

2

3

3

3

2

3

2.4
3

2

3

2 
The self-assessment questionnaire in water control is in high performance with grade point 3 . Besides the independent water system, environmental surrounding field cultivations (forest and rural areas) identically with a catcher water area and far away from chemical or household waste. Other, the advantage of this system are considered a critical point to eliminate contaminations potential which is the consumer may not be use hygiene water for washing, preferably eat directly (James, 2006; Kavyashri, et al., 2016; Huang, et al., 2016)

\section{Concussion}

These findings demonstrated and confirmed, all of the local farms have medium performance in application of good agriculture practice. Particularly, preventing the Salmonella spp and maintaining the hygiene attribute through food safety activities and following the GAP criteria. However, the assurance activities are still limited information about the improving program and lack of core activities in laboratory checking system. Other, local government and cooperatives collaborative support is considered as a credit to improving the comprehensive aspect (knowledge, funding support) of food safety.

\section{References}

Abadias, M., Usall, J., Anguera, M., Solsona, C., \& Viñas, I. (2008). Microbiological quality of fresh, minimally-processed fruit and vegetables, and sprouts from retail establishments. International Journal of Food Microbiology, 123(1-2), 121-129.

Ackers, M. L., Mahon, B. E., Leahy, E., Goode, B., Damrow, T., Hayes, P. S., et al. (1998). An outbreak of Escherichia coli O157:H7 infections associated with leaf lettuce consumption. The Journal of Infectious Diseases, 177(6), 1588e1593.

AOAC International. (2002). Official methods of analysis of AOAC international (17thed.) Gaithersburg.

APHA. (1998). Standard methods for the examination of water and wastewater (20th ed.). Washington: American Public Health Association, American Water Works Association, Water Environmental Federation.

Beuchat, L. R. (1996). Pathogenic microorganisms associated with fresh produce. Journal of Food Protection, 59(2), 204e216.

Codex Alimentarius. (2003). Code of hygienic practice for fresh fruits and vegetables. Cometti, N. N., Matias, G. C. S., Zonta, E.,

Delaquis, P., Bach, S., \& Dinu, L. D. (2007). The behavior of Escherichia coli O157: H7 in leafy vegetables. Journal of Food Protection, 70(8), 1966 - 1974.

Fong, T. T., Mansfield, L. S., Wilson, D. L., Schwab, D. J., Molloy, S. L., \& Rose, J. B. (2007). Massive microbiological groundwater contamination associated with a waterborne outbreak in Lake Erie, South Bass Island, Ohio. Environmental Health Perspectives, 115(6), 856 - 864.

Harris, L. J., Berry, E. D., Blessington, T., Erickson, M., Jay-Russell, M., Jiang, X., et al. (2013). A framework for developing research protocols for evaluation of microbial hazards and controls during production that pertain to the application of untreated soil amendments of animal origin on land used to grow produce that may be consumed raw. Journal of Food Protection, 76(6), $1062-1084$. 
Islam, M., Doyle, M. P., Phatak, S. C., Millner, P., \& Jiang, X. (2005). Survival of Escherichia coli O157:H7 in soil and on carrots and onions grown in fields treated with contaminated manure composts or irrigation water. Food Microbiology, 22(1), $63-70$.

ISO, International Standard. (2010). Microbiology of food and animal feeding stuffs: Horizontal method for the detection of Salmonella spp (1st ed.).

Jacxsens, L., Devlieghere, F., \& Uyttendaele, M. (2009). Quality management systems in the food industry (2nd ed.). Sofia, Bulgaria: St Kliment Ohridski University Press.

Jacxsens, L., Kussage, K., Luning, P. A., Spiegel, Van der, M., Devlieghere, F., et al. (2009). A microbial assessment scheme to measure microbial performance of food safety management systems. International Journal of Food Microbiology, 134(1 - 2), 113 - 125.

Jacxsens, L., Uyttendaele, M., Devlieghere, F., Rovira, J., Oses Gomez, S., \& Luning, P. A. (2010). Food safety performance indicators to benchmark food safety output of food safety management systems. International Journal of Food Microbiology, 141, S180 - S187.

James, J. (2006). Microbial hazard identification in fresh fruits and vegetables. Dublin, Ireland: Wiley-Interscience.

Johannessen, G. S. (2005). Use of manure in the production of organic lettuce: Risk of transmission of pathogenic bacteria and bacteriological quality of the lettuce. Oslo, Belgic: Norwegian Sch ool of Veterinary Science.

Kavyashri, V.V., Anil Pappachan, A.S. Padmaja, N. Nagaraju, and K.T. Rangaswamy. (2016). Biological and molecular characterization of cucumber mosaic virus isolate causing severe mosaic in Gherkin (Cucumis Anguria L.) in India. Journal of Pure and Applied Microbiology, 10 (3) p. $2089-3008$

Kirezieva, K., Nanyunja, J., Jacxsens, L., Uyttendaele, M., Van der Vorst, J., \& Luning, P. (2013). Context factors affecting design and operation of food safety management systems in the fresh produce chain. Trends in Food Science and Technology, 32(2), 108 - 127.

Luning, P. A., Marcelis, W. J., van Boekel, M. A. J. S., Rovira, J., Uyttendaele, M., \& Jacxsens, L. (2011). A tool to diagnose context riskiness in view of food safety activities and microbiological safety output. Trends in Food Science and Technology, 22(1), S67 - S79.

MAFF, The Ministry of Agriculture, Fisheries and Food. (2016). Statistical yearbook 2015.

Md. Sajjad Alam, Farahnaaz Feroz, Hasibur Rahman, Kamal Kanta Das, Rashed Noor, (2015) "Microbiological contamination sources of freshly cultivated vegetables", Nutrition \& Food Science, Vol. 45 Issue: 4, pp.646-658

Michaels, B., \& Todd, E. (2005). Food worker personal hygiene requirements during harvesting, processing, and packaging of plant products. In J. James (Ed.), Microbial hazard identification in fresh fruit and vegetables (pp. 115e153). Nova Jersey, EUA: John Wiley \& Sons, Inc.

Millner, P. (2003). Composting: improving on a time-tested technique. Agricultural Research, 51(8). Moyne, A. L., Sudarshana, M. R., Blessington, T., Koike, S. T., Cahn, M. D., \& Harris, L. J. (2011). Fate of Escherichia coli O157:H7 in field-inoculated lettuce. Food Microbiology, 28(8), $1417-1425$.

Uyttendaele, M., Jacxsens, L., Luning, P., Van der Vorst, J., Devlieghere, F., \& Leemans, R. (2010). Simulation modeling and risk assessment as tools to identify the impact of climate change on microbiological food safety: the case study of fresh produce supply chain. Food Micro, Abstracts. Presented at the 22nd International ICFMH Symposium (Food Micro 2010) : Microbial behavior in the food chain. 
Nieto-Montenegro, S., Brown, J. L., \& LaBorde, L. F. (2008). Development and assessment of pilot food safety educational materials and training strategies for Hispanic workers in the mushroom industry using the Health Action Model. Food Control, 19(6), 616 - 633.

Okello, J. J., \& Swinton, S. M. (2007). Compliance with international food safety standards in Kenya's green bean industry: comparison of a small- and a largescale farm producing for export. Review of Agricultural Economics, 29(2), 269 - 285.

Olaimat, A. N., \& Holley, R. A. (2012). Factors influencing the microbial safety of fresh produce: a review. Journal of Food Protection, 32(1), 1 - 19.

Oliveira, M., Usall, J., Viñas, I., Anguera, M., Gatius, F., \& Abadias, M. (2010). Microbiological quality of fresh lettuce from organic and conventional production. Food Microbiology, 27(5), $679-684$.

Oses, S., Luning, P., Jacxsens, L., Santillana, S., Jaime, I., \& Rovira, J. (2012). Food safety management system performance in the lamb chain. Food Control, 25(2), $493-500$.

Umesha, S., Manukumar, H. M., Chandrasekhar, B., Shivakumara, P., Shiva Kumar, J., Raghava, S. , Avinash, P., Shirin, M. , Bharathi, T. R., Rajini, S. B., Nandhini, M. , Vinaya Rani, G. g., Shobha, M. and Prakash, H. S. (2017), Aflatoxins and food pathogens: impact of biologically active aflatoxins and their control strategies. J. Sci. Food Agric., 97: 1698-1707.

Xinqi Huang, Liangliang Liu, Teng Wen, Jinbo Zhang, Fenghe Wang, and Zucong Cai. (2016). Changes in the soil microbial community after reductive soil disinfestation and cucumber seedling cultivation. Applied Microbiology and Biotechnology. 100 (12) pp 5581-5593. 